\title{
EAHP European Statements baseline survey 2015: methodology
}

\author{
Jonathan Underhill, Nicholas Gibbons
}

Keele Centre for Medicines Optimisation, Keele University School of Pharmacy, Keele, UK

\section{Correspondence to}

Jonathan Underhill, Keele Centre for Medicines

Optimisation, Keele University School of Pharmacy, Keele ST5 5BG, UK; j.l.underhill@keele. ac.uk

Received 19 January 2016 Revised 26 January 2016 Accepted 27 January 2016

\section{SLinked}

- http://dx.doi.org/10.1136/ ejhpharm-2016-000892 - http://dx.doi.org/10.1136/ ejhpharm-2016-000889

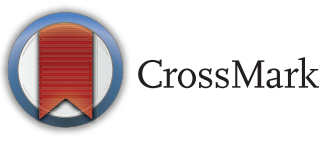

To cite: Underhill J, Gibbons N. Eur J Hosp Pharm 2016:23:65-68.

\section{ABSTRACT}

The European Statements of Hospital Pharmacy are a set of hospital pharmacy practice standards published by the European Association of Hospital Pharmacists (EAHP) for European health systems to ensure safe, effective and optimal use of medicines in collaboration with multidisciplinary teams. Keele University was commissioned to conduct a survey among European hospital pharmacists to establish a baseline to assess awareness of the Statements and to identify any barriers to their implementation. The survey was conducted from January 2015 to March 2015, spanning 16 languages and 34 countries. The survey consisted of three sections:

- Section A: general questions about the participant's hospital pharmacy, such as workforce skill-mix and number of beds served;

- Section B: questions about the current activity of pharmacists around each statement;

- Section C: questions about the hospital's readiness and ability to implement the statements.

In section $B$, a value was allocated to each response to rate the degree to which they were able to comply with each statement (where $1=$ never able to comply, $5=a l$ ways complied). In section C, they were asked to what degree they agreed with the question ( 1 for strongly disagree, 5 for strongly agree). A response of 1 or 2 was deemed to indicate some difficulty in complying with that statement-a 'negative response'. Where this was the case, the participant was given the option to provide a free text response explaining the reasons for this difficulty.

The full results are discussed in a subsequent paper (see page 69). Overall, the response rate was $18 \%$, but the variation in this was marked. 22 of the 34 countries had a response rate of over $30 \%$. The methods used for this survey results informs the methodology and scope of future EAHP surveys

Recommendations for future surveys are as follows:

- Keep the survey short and easy to complete (to within $20 \mathrm{~min}$ );

- Specifically enquire for each question if capacity and capability are the key barriers to implementation;

- Construct survey response options for each question to identify barriers other than capacity and capability;

- Identify the key drivers for change in countries where implementation has occurred or is occurring;

- Compile better intelligence about the number of surveys sent out in the first place (as those countries with a low response rate sent out lots of invites to participate which may be unrealistic);

- A named person (country coordinator) to send out invite survey link;

- Weekly reminders should be sent out by the named person (country coordinator).

\section{INTRODUCTION AND BACKGROUND}

The European Statements of Hospital Pharmacy are a set of hospital pharmacy practice standards compiled and published by the European Association of Hospital Pharmacists (EAHP) for European health systems to ensure safe, effective and optimal use of medicines in collaboration with multidisciplinary teams. ${ }^{1}$

The statements were formulated following an 18-month review process, which included two rounds of online Delphi consultation with EAHP's 34 member country associations and patient and healthcare professional organisations and a 'World Café.' As outlined by Horák et $a l^{3}$ in their report on the future of the EAHP survey, implementation of the Statements remains a challenge. Generally, the biggest challenges in implementing the Statements are perceived to be around the varying levels of practice, the different healthcare systems and problems with staffing (capacity and capability). In order to facilitate better implementation of the Statements, it is essential to capture a baseline of where different countries are now in relation to each Statement and then measure their progress on a regular basis. Based on previous feedback and the Summit, EAHP decided to change its data collection tool, the EAHP survey, by designing a shorter annual survey, optimising data collection while minimising workload for survey respondents. The primary focus of the annual survey is to identify the barriers to the implementation of the Statements.

The Keele Centre for Medicines Optimisation at Keele University was commissioned in September 2014 to conduct a survey among European hospital pharmacists to establish a baseline to assess awareness of the Statements and to identify any barriers to their implementation.

\section{METHOD}

\section{Survey development}

The survey was drafted following a meeting of the EAHP Survey Group facilitated by two external consultants (CMcK and JU) where the pros and cons of several question styles were discussed and agreed. The initial draft of the questions were commented on by the EAHP Survey Group and then sent to each country coordinator for comment. These comments were incorporated to produce the final version.

It was agreed by the EAHP Survey Group that the survey should only ask questions based on statements that had a resonance at an individual hospital level. The remaining statements which could only be responded to at a national level were statements 1.2, 1.4, 4.7, 5.1, 6.1 and 6.5. The EAHP conducted a separate survey for these statements, with the results of the questions relating to those statements reported as an appendix to the overall report, published on the EAHP website. 
The survey was created by NG using the online survey software SurveyMonkey. This allowed the survey to incorporate a variety of question formats and necessary logic, while incorporating EAHP branding and logos.

\section{Survey design and format}

The primary focus of the survey was to identify the barriers in the implementation of the European Statements of Hospital Pharmacy. To achieve this aim, the survey (see appendix 1) consisted of three sections:

- Section A: general questions about the participant's hospital pharmacy, such as workforce skill-mix and number of beds served.

- Section B: questions about the current activity of pharmacists around each statement.

- Section C: questions about the hospital's readiness and ability to implement the statements.

In section B, the pharmacists who participated in the survey were asked to rate the degree to which they were able to comply with each statement. A value was allocated to each response using a scale of $1-5$, where 1 indicated that they were never able to comply with the statement, while 5 indicated that they always complied with the statement. In section $C$, they were asked to what degree they agreed with the question and the same Likert scale was used (1 for strongly disagree, 5 for strongly agree).

For the purposes of identifying those statements where the barriers to implementation were greatest, a response of 1 or 2 was deemed to indicate some difficulty in complying with that statement-a 'negative response'. Where this was the case, the participant was given the option to provide a free text response explaining the reasons for this difficulty.

The survey was designed to only prompt for a free text response when the participant gave a negative response. By doing this, the logistical burden of dealing with vast amounts of free text (both translation and analysis time) was minimised, while still enabling the barriers to be identified by responders. For these reasons, it was also decided that all free text responses should be limited to 200 characters, prompting participants to answer concisely. Participants were also given the option to email a full response if they felt the need to.

Translation of the survey into 15 languages other than English was facilitated by EAHP staff using country coordinators to check the translated text for their own country. The translated survey text was then sent back to Keele and entered into SurveyMonkey. The same process was used for the translation of free text from the completed surveys.

\section{COMPLETION OF THE SURVEY}

The survey was conducted from January 2015 to March 2015, spanning 16 languages and 34 countries. Access to the survey was through a web-link, meaning that anybody with access to the link could complete the survey. NG sent a link to the survey and a list of unique codes to an EAHP coordinator who distributed the survey to the recipients and could track responses. In cases where a country did not wish to share the emails of their pharmacists, the EAHP sent the link and codes to a coordinator within the country to distribute themselves.

When the user started the survey they were asked to input their unique code, which allowed the survey administrators to check that only people invited to the survey were responding. This also enabled monitoring of response rates to the survey, where weekly reminders could then be sent to those who had not yet replied.

\section{SURVEY ANALYSIS}

When the survey closed, there were a total of 1094 responses, the results of which were exported from SurveyMonkey by NG for further analysis and reporting. For each country, the percentage of 'negative responses' was calculated for each question with the five questions receiving the highest proportion of negative responses identified for each country. The advantage of using this method instead of the mean meant that the views of each country were considered equally, despite the fact that there was a large variation in the number of responses received for each country.

\section{SURVEY RESPONSE RATES}

The response rates of completed surveys are listed in table 1, broken down by country. If an incomplete survey was submitted, the quantitative data were not used in the results, although any free text responses were still incorporated. For the majority of cases, the country's coordinator provided a list of emails for person they wished to include in the survey, a unique survey code was generated for each person and the EAHP distributed the survey. However, the countries highlighted in red (table 1) used a different distribution method and were instead provided a list of unique codes to allocate and distribute to people themselves (table 1 ).

Table 1 Response rates to the survey by country

\begin{tabular}{|c|c|c|c|}
\hline Country & Responses & Requests & Response rate (\%) \\
\hline Iceland & 1 & 1 & 100 \\
\hline Denmark & 7 & 8 & 88 \\
\hline Croatia & 32 & 39 & 82 \\
\hline Slovenia & 19 & 28 & 68 \\
\hline Malta & 4 & 6 & 67 \\
\hline Norway & 21 & 32 & 66 \\
\hline Hungary & 67 & 105 & 64 \\
\hline Serbia & 42 & 67 & 63 \\
\hline Czech & 57 & 94 & 61 \\
\hline Macedonia & 19 & 33 & 58 \\
\hline Ireland & 39 & 73 & 53 \\
\hline Luxembourg & 3 & 6 & 50 \\
\hline Austria & 22 & 45 & 49 \\
\hline Switzerland & 28 & 58 & 48 \\
\hline Bosnia & 10 & 21 & 48 \\
\hline Slovakia & 29 & 61 & 48 \\
\hline Sweden & 16 & 34 & 47 \\
\hline Estonia & 10 & 22 & 45 \\
\hline Romania & 28 & 63 & 44 \\
\hline UK & 58 & 154 & 38 \\
\hline Netherlands & 29 & 82 & 35 \\
\hline Greece & 35 & 115 & 30 \\
\hline Finland & 24 & 89 & 27 \\
\hline Belgium & 43 & 169 & 25 \\
\hline Germany & 95 & 388 & 24 \\
\hline Portugal & 23 & 118 & 19 \\
\hline Spain & 45 & 250 & 18 \\
\hline Bulgaria & 11 & 66 & 17 \\
\hline Lithuania & 2 & 15 & 13 \\
\hline Latvia & 6 & 47 & 13 \\
\hline Turkey & 47 & 543 & 9 \\
\hline France & 152 & 1888 & 8 \\
\hline Poland & 35 & 600 & 6 \\
\hline Italy & 35 & 606 & 6 \\
\hline Total & 1094 & 5926 & 18 \\
\hline
\end{tabular}




\section{DISCUSSION AND RECOMMENDATIONS FOR FUTURE SURVEYS}

The results of the baseline survey are discussed in a subsequent paper (see page 69), and how this informs the EAHP implementation strategy the subject of another further paper (see page 76).

The method used for this survey provides a good overall baseline picture of where the different member countries are in relation to each of the Statements. Overall, the response rate was $18 \%$, but the variation in this was marked. Of the 34 countries, $22 \mathrm{had}$ a response rate of over $30 \%$ which is in line with the response rate seen for previous EAHP surveys. Those countries where lots of surveys were sent out were the ones who tended to have lower response rates. This should inform the methodology used for future surveys where better targeting of the survey is recommended to increase the percentage response rate.

The survey was designed to ease the burden of translating large amounts of free text responses by only asking for free text responses if the participant responded negatively to a question, and to limit the response to 200 characters. Despite this, translation of free text still proved to be a significant task.

One solution to this problem for future surveys would be to identify key barriers to implementation from the results of this survey (eg, capacity and capability) and provide future survey participants the ability to select options from a list of key barriers to implementation. The participants would then only need to provide a free text response if they encountered barriers not in the list of options.

Recommendations for future surveys:

- Keep the survey short and easy to complete (to within $20 \mathrm{~min})$

- Specifically enquire for each question if capacity and capability are the key barriers to implementation

- Construct survey response options for each question to identify barriers other than capacity and capability

- Identify the key drivers for change in countries where implementation has occurred or is occurring

- Compile better intelligence about the number of surveys sent out in the first place (as those countries with a low response rate sent out lots of invites to participate which may be unrealistic)

- A named person (country coordinator) to send out invite survey link

- Weekly reminders should be sent out by the named person (country coordinator)

\section{What this paper adds}

What is already known on this subject

- The European Statements of Hospital Pharmacy, expressing commonly agreed objectives which every European health system should aim for in the delivery of hospital pharmacy services, were prepared and approved in 2014.

- Implementation of the European Statements of Hospital Pharmacy is a key priority for hospital pharmacy.

- There were no specific data describing the level of implementation and main barriers to this.

\section{What this study adds}

- The baseline survey aims to identify the barriers and drivers of implementation of the Statements.

- The methods used for this survey results informs the methodology and scope of future European Association of Hospital Pharmacists surveys.

Acknowledgements EAHP Survey Working Group: Petr Horák, Joan Peppard, Juraj Sýkora, Tajda Miharija Gala, Leonidas Tzimis, Ercan Celikkayalar Adil and Vera Pires. Ms Jennie De Greef and EAHP staff. EAHP member association presidents and national survey coordinators. Cheryl McKay (CMcK), managing director, Kayhill Consulting (http:// www.kayhillconsulting.com/).

Contributors The survey group, NG and JU were involved in planning of the study, design of the questionnaire and conducting of survey. CMcK was involved in the initial planning of the survey. NG set up the online form, along with respective language mutations. NG, JU and PH were involved in data evaluation and statistics. JU and NG drafted the article and revised for content; JU had final approval of the version to be published.

Competing interests None declared.

Provenance and peer review Not commissioned; internally peer reviewed.

\section{REFERENCES}

1 The European Statements of Hospital Pharmacy. Eur J Hosp Pharm 2014;21:256-8.

2 Maskrey N, Underhill J. The European Statements of Hospital Pharmacy: achieving consensus using Delphi and World Café methodologies. Eur I Hosp Pharm 2014;21:264-6.

3 Horák P, Peppard J, Sykora J, et al. EAHP survey and European Statements of Hospital Pharmacy—can we achieve a perfect match? Eur J Hosp Pharm 2014;21:291-3.

\section{APPENDIX 1}

The table below shows all of the questions asked in the survey regarding the 44 European Statements of Hospital Pharmacy, as well as the overall percentage of participants who gave a 'negative response' to the question.

Questions where more than $25 \%$ of participants gave a negative response have been highlighted in red.

\section{EAHP Survey Questions}

Section 1: Introductory Statements and Governance

S11 The pharmacists in our hospital work routinely as part of multidisciplinary team ( $41 \%$ of all responses were negative)

S13 Our hospital is able prioritise hospital pharmacy activities according to agreed criteria ( $23 \%$ of all responses were negative)

S15 The pharmacists in our hospital are engaged in the supervision of all steps of all medicine use processes ( $24 \%$ of all responses were negative)

S16 At least one pharmacist from our team is a full member of the Drug and Therapeutics Committee (11\% of all responses were negative)

S162 The pharmacists in our hospital take the lead in coordinating the activities of the Drug and Therapeutics Committees (26\% of all responses were negative) S17 The pharmacists in our hospital are involved in the design, specification of parameters and evaluation of ICT used within medicines processes (27\% of all responses were negative) 
Continued

\section{EAHP Survey Questions}

\section{Section 2: Selection, Procurement and Distribution}

S21 Our hospital has clear processes in place around the procurement of medicines (4\% of all responses were negative)

S212 Were hospital pharmacists involved in the development of these? ( $6 \%$ of all responses were negative)

S22 The pharmacists in our hospital take the lead in developing, monitoring, reviewing and improving medicine use processes and the use of medicine-related technologies (19\% of all responses were negative)

S23 The pharmacists in our hospital coordinate the development, maintenance and use of our formulary (16\% of all responses were negative)

S24 Procurement of non-formulary medicines in our hospital is done to a robust process ( $9 \%$ of all responses were negative)

S25 The pharmacy in our hospital has contingency plans for medicines shortages (30\% of all responses were negative)

S26 The pharmacy in our hospital takes responsibility for all medicines logistics, including for investigational medicines (8\% of all responses were negative)

S27 Our hospital has a policy for the use of medicines brought into the hospital by patients ( $28 \%$ of all responses were negative)

S272 Were pharmacists involved in producing this policy? (16\% of all responses were negative)

Section 3: Production and Compounding

S31 The pharmacists in our hospital check if a suitable product is commercially available before we manufacture or prepare a medicine (8\% of all responses were negative)

S32 When medicines require manufacture or compounding, we either produce them in our hospital pharmacy or we outsource to an approved provider ( $9 \%$ of all responses were negative)

S33 The pharmacists in our hospital undertake a risk assessment to determine the best practice quality requirements before making a pharmacy preparation (16\% of all responses were negative)

S34 The pharmacy in our hospital has an appropriate system in place for the quality assurance of pharmacy prepared and compounded medicines ( $24 \%$ of all responses were negative)

S342 The pharmacy in our hospital has an appropriate system in place for the traceability of pharmacy prepared and compounded medicines (17\% of all responses were negative)

S35 Our hospital has appropriate systems in place for the preparation and supply of hazardous medicines (26\% of all responses were negative)

S36 Our hospital has written procedures that ensure staff are appropriately trained to reconstitute or mix medicines in a patient care area (25\% of all responses were negative)

S362 Were pharmacists involved in approving these procedures? (28\% of all responses were negative)

Section 4: Clinical Pharmacy Services

S41 The pharmacists in our hospital play a full part in shared decision-making on medicines, including advising, implementing and monitoring medication changes (31\% of all responses were negative)

S42 All prescriptions in our hospital are reviewed and validated as soon as possible by a pharmacist ( $37 \%$ of all responses were negative)

S43 The pharmacists in our hospital have access to the patients' health record (34\% of all responses were negative)

S432 The pharmacists in our hospital can document their clinical interventions into the patients' health record (44\% of all responses were negative)

S434 We analyse clinical pharmacy interventions to inform quality improvement plans (14\% of responses were negative)

S44 The pharmacists in our hospital enter all medicines used onto the patient's medical record on admission (71\% of all responses were negative)

S442 The pharmacists in our hospital reconcile medicines on admission ( $22 \%$ of all responses were negative)

S444 The pharmacists in our hospital assess the appropriateness of all patients' medicines, including herbal and dietary supplements (9\% of all responses were negative)

S45 The pharmacists in our hospital contribute to the transfer of information about medicines when patients move between and within healthcare settings ( $56 \%$ of all responses were negative)

S46 The pharmacists in our hospital ensure patients and carers are offered information about their medicines in terms they can understand (36\% of all responses were negative)

Section 5: Patient Safety and Quality Assurance

S52 Our hospital has appropriate strategies to detect errors and identify priorities for improvement in medicines use processes (21\% of all responses were negative)

S522 Were pharmacists involved in approving these procedures? (18\% of all responses were negative)

S53 Our hospital uses an external quality assessment accreditation programme to assure our medicines use processes (48\% of all responses were negative)

S532 Our hospital acts on these reports to improve the quality and safety of our medicines use processes (29\% of all responses were negative)

S54 The pharmacists in our hospital report adverse drug reactions ( $24 \%$ of all responses were negative)

S542 The pharmacists in our hospital report medication errors (32\% of all responses were negative)

S55 The pharmacists in our hospital use evidence-based approaches to reduce the risk of medication errors ( $21 \%$ of all responses were negative)

S552 Our hospital pharmacy uses computerised decision support to reduce the risk of medication errors (39\% of all responses were negative)

S56 Our hospital has appropriate procedures in place to identify high-risk medicines and minimise risk from their use (19\% of all responses were negative)

S562 Are pharmacists involved in implementing these procedures? (7\% of all responses were negative)

S57 The medicines administration process in our hospital ensures that transcription steps between the original prescription and the medicines administration record are eliminated (31\% of all responses were negative)

S58 Our patient's health records accurately record all allergy and other relevant medicine-related information (12\% of all responses were negative)

S59 The pharmacists in our hospital ensure that the information needed for safe medicines use is accessible at the point of care (13\% of all responses were negative)

S510 Medicines in our hospital are packaged and labelled to assure they are safely optimised for administration (11\% of all responses were negative)

S511 Medicines dispensed by our pharmacy are traceable ( $12 \%$ of all responses were negative)

Section 6: Education and Research

S62 The pharmacists in our hospital are able to demonstrate their competence to perform their roles (7\% of all responses were negative)

S63 Pharmacists in our hospital are able to engage in relevant educational opportunities ( $5 \%$ of responses were negative)

S64 The pharmacists in our hospital routinely publish hospital pharmacy practice research ( $51 \%$ of all responses were negative) 